\title{
LES MIGRATIONS SELECTIVES. UNE REPONSE A LA CRISE?
}

\author{
André Fischer*
}

Pendant toute la première phase de l'Aménagement du Territoire en France, phase au cours de laquelle l'industrie joue un róle majeur dans l'aménagement, la politique territoriale dominante est celle de la décentralisation aux profits des espaces périphériques attardés ou en crise. Le principe fondamental sur lequel s'appuie cette politique territoriale est celui de l'aide à la dispersion géographique des investissements, donc des emplois, pour permettre aux actifs de "travailler et vivre au pays"

En 1979, dans un discours prononcé à Lyon, le Premier Ministre d'alors déclare que, compte tenu de la crise, il est désormais temps de favoriser la mobilité des actifs vers les emplois, donc, pour l'essentiel, vers les métropoles et grandes agglomérations où se créent la plupart des emplois nouveaux.

$\mathrm{Vu}$ à travers le prisme des mouvements migratoires, le changement dans l'action territoriale des pouvoirs publics est donc considérable puisqu'on passe d'une politique de dispersion spatiale des capitaux à une politique, rarement exprimée de façon explicite, de concentration géographique des emplois aux profits des centres urbains les plus dynamiques. Bien entendu, ce changement affecte en priorité la partie de la population active la plus sensible aux mutations qualitatives de l'activité économique, c'est à dire les actifs les plus qualifiés.

Très rapidement, au cours des années quatre-vingt, des migrations sélectives d'actifs se développent, en relation avec les phénomènes de ségrégation qualitative des activités introduits dans la plupart des espaces géographiques par l'innovation technologique et son corollaire la métropolisation. Pour les entreprises comme pour les espaces-territoires, les migrations sélectives deviennent un instrument d'ajustement aux effets de la crise.

Les réflexions qui suivent, à propos de la compréhension et de l'explication de ces migrations sélectives, se fondent sur les tendances dominantes qui caractérisent l'évolution contemporaine des activités industrielles.

\section{Le Changement de systeme productif et ses efects}

Entre les années soixante-dix et quatrevingt on assiste à un déclin rapide du système de production fordiste et à un essor non moins rapide du système de production flexible. Les mutations qui ont accompagné ce changement se sont particulièrement manifestées dans les domaines suivant: les structures internes des entreprises, la nature et le degré de qualification des emplois, la localisation géographique des établissements, la répartition territoriale des diverses catégories d'actifs. Pour comprendre pourquoi la mobilité des actifs s'accentue et pourquoi elle devient de plus en plus sélective, il faut entrer dans le détail des systèmes de production et de leur évolution.

La grande dimension, des unités économiques et techniques, la concentration fonction-

\footnotetext{
* Professor Emérito
}

Universidade de Paris 1 Panthéon -Sorbonne 
nelle et l'internalisation des fonctions, le recours aux bienfaits de l'Etat -providence ... sont quelques-uns des dogmes traditionnels du système de production fordiste. L'importance des grandes entreprises et surtout des grands établissements se traduit par une longue tradition de relative înertie spatiale (le transfert spatial de l'établissement est souvent l'ultime mesure d'ajustement mise en oeuvre pour sauver l'activité, ce qui a fait croire à la pérennité des facteurs de localisation !). Bien avant les années quatre-vingt, la recherche de solutions aux problèmes du coût du travail a poussé les entreprises à utiliser des maind'oeuvre moins coûteuses, d'où l'essor du double mouvement géographique de la délocalisation (vers les pays du Tiers Monde dans le cadre de la Division Internationale du Travail) et de la décentralisation (dans le cadre des politiques nationales d'aménagement en faveur des régions périphériques). Ces deux mouvements vont s'accélérer considérablement avec la crise du système fordiste et l'émergence rapide du système de production flexible.

Le système flexible se traduit, en effet, par quelques changements radicaux tels que le passage d'une logique dominante à caractère industriel à une logique dominante de type financier, l'essor des dogmes de la petite dimension de la spécialissation fonctionnelle de l'externalisation des fonctions du développement des relations horizontales avec le milieu d'implantation... Mais surtout, le système de production flexible impose un changement radical en matière d'exigences de fonctionnement des entreprises et des établissements, c'est ce changement, lié à l'innovation technologique et à l'adoption des technologies nouvelles, qui va nous permettre de mieux comprendre le problème général des migrations sélectives et de leur signification.

L'éclatement structurel et fonctionnel des firmes est la première manifestation majeure du nouveau système de production: le premier s'exprime par la multiplication des petites unités spécialisées; le second conduit à une distinction croissante faite entre les activités du péri-produc- tif amont, les activités de production stricto sensu, les activités du péri-productif aval. Dès lors, les processus de production et leur relation à l'espace géographique changent considérablement. L'essor des processus de production discrets, caractéristique de l'innovation technologique, se traduit, pour les grandes entreprises à établissements multiples, par une généralisation de la disjonction fonctionnelle, de la segmentation technique du travail, de la division sociale et spatiale du travail. Les établissements étant spécialisés, au plan fonctionnel, leur main-d'oeuvre tend à une certaine unicité, en rapport avec les technologies mises en oeuvre, tandis que se développent de nouvelles relations avec le milieu d'implantation à travers la sous-traitance, l'essaimage, les synergies, les partenariats... mais aussi à travers le recours systématique aux externalités de service offertes par le milieu local ou régional. Exprimés en termes de tendances dominantes, les résultats géographiques de cet éclatement interne des firmes sont aujourd'hui bien connus: d'une part, une mobilité spatiale considérablement accrue (et facilitée par la planification décentralisée et l'ubiquité des externalités de service); d'autre part, une concentration géographique des fonctions et des activités les plus sophistiquées dans les niveaux supérieurs des hiérarchies urbaines régionales et nationales alors que les fonctions et activités banales, souvent fortement standardisées, ont surtout tendance à se disperser dans les espaces périphériques disposant de réservoirs de main-d'oeuvre bon marché. En d'autres termes, la contradiction entre ces deux mouvements géographiques ayant des bases qualitatives (de fonctions, d'activités, de maind'oeuvre, de technologies), on peut dire que le système de production flexible génère d'importantes ségrégations qualitatives dans l'espace géographique.

L'éclatement des marchés du travail et de l'emploi constitue la seconde manifestation majeure de la mise en place du nouveau système de production. L'innovation technologique et les technologies nouvelles ont, tout à la fois, rendu 
obsolètes la majorité des savoir-faire traditionnels, provoqué la déqualification d'un grand nombre d'emplois et généré l'apparition de multiples métiers nouveaux traduisant une évolution globale du travail vers plus de tertiairisation, plus d'intellectualisation, plus de qualification. Dans tous les vieux bassins industriels d'Europe occidentale et dans la plupart des branches d'activité traditionnelles, cette évolution a provoqué la disparition de dizaines de milliers d'emplois; partout, la nécessité s'est imposée de requalifier ou de qualifier les hommes en multipliant les moyens et les structures de formation. Simultanément, le nouveau système de production s'efforce de rendre également plus flexible le travail, donc les emplois et les hommes. Pour obtenir cette flexibilisation du travail, "l'arme absolue" développée par le système est celle de la précarisation du travail, sous toutes ses formes: volants de chómage, viviers de main-d'oeuvre hautement qualifiée, réservoirs de main-d'oeuvre à faible qualification, emplois temporaires, travail à temps partiel, contrats à durée limitée, interim... etc. Le résultat de la combinaison entre le renouvellement incessant des technologies et la précarisation généralisée du travail est clair: les hommes doivent satisfaire tout à la fois à l'obligation de mobilité professionnelle (il faut ètre apte à changer plusieurs fois d'emploi au cours de sa vie d'actif) ainsi qu'à l'obligation de mobilité spatiale (il faut être apte à se déplacer vers les emplois offerts). Cette double mobilité va être, implicitement pour les pouvoirs publics et explicitement pour les entreprises, utilisée, par l'intermédiaire des migrations sélectives, pour réaliser l'un des grands objectifs des politiques d'aménagement: l'ajustement spatial et fonctionnel entre les bassins d'emplois (l'offre de travail) et les bassins de main-d'oeuvre (la demande de travail).

Un bilan des effets réciproques de l'éclatement interne des grandes entreprises et de l'éclatement du travail fait apparaitre, dans l'espace géographique, un résultat non négligeable: les entreprises ont désormais tendance à réaliser l'adéquation la plus étroite possible entre la technologie qu'elles veulent mettre en oeuvre et le niveau de qualif ication de la force de travail qu'elles veulent employer; et cette adéquation se réalise de plus en plus selon le principe défini par P. AYDALOT: "Une fonction, une technologie, une main-d'oeuvre, un lieu" (AYDALOT. 1983). Dès lors, on comprend mieux, d'une part, l'importance des stratégies spatiales des entreprises, d'autre part, l'importance du phénomène de migrations sélectives des actifs.

\section{L'ajustement des espaces geographiques}

Les activités de haute technologie ont des exigences spécifiques en matière de niveau de qualification de la main-d'oeuvre, d'information, d'externalités de service, de ressources disponibles, de relations au milieu ... etc.. Globalement, ces exigences de fonctionnement représentent une "combinatoire" originale qui ne saurait exister partout dans l'espace géographique mais qui constitue un facteur très discriminant sur le plan qualitatif. Par leur contenu et leur offre, certains espaces sont plus attractifs que d'autres, d'où les différences qualitatives en matière d'activités et de main-d'oeuvre employée.

Par ailleurs, pour ce qui concerne plus spécifiquement le cas français, il est certain que le développement assez rapide de la décentralisation administrative, de la planification décentralisée et du transfert territorial de compétences, au cours des années quatre-vingt, a eu pour conséquence directe une accélération de la mobilité spatiale de l'offre d'externalités de service, ce qui a fortement pesé sur les rapports entre les phénomènes de concentration spatiale/dispersion spatiale sélectives. En d'autres termes, il en est résulté une accentuation des ségrégations qualitatives dans l'espace géographique.

Avec le système de production flexible, les territoires sont de plus en plus marqués par le jeu contradictoire des mouvements de concentration spatiale des fonctions et activités sophistiquées et les mouvements de dispersion spatiale des fonctions et activités banales; les migrations 
interrégionales ou internationales des actifs sont, bien entendu, le fidèle reflet de ces tendances géographiques lourdes. On peut invo quer plusieurs facteurs explicatifs pour bien comprendre le poids de ces tendances. Les différentes catégories de fonctions n'ont pas les mèmes exigences donc pas les mèmes comportements dans l'espace géographique; et les fonctions liées au péri-productif, celles qui exigent le plus de recours aux externalités, sont de plus en plus nombreuses et diversifiées avec l'innovation permanente. Les coûts de localisation sont très variables et peuvent entrainer, en raison de leur importance, des discriminations dans les implantations d'activités. Les coûts salariaux et le coût de la reproduction de la force de travail peuvent être d'un poids très lourd dans le coūt total de production; dans ce cas, il ne peut ètre question de s'implanter dans des milieux où les coûts de localisation sont trop élevés par rapport au niveau de la valeur ajoutée générée par les activités concernées. Deux facteurs exogènes concourent également au renforcement sélectif des mouvements de concentration spatiale: d'une part, le caractère cumulatif des effets réciproques des accumulations de pouvoirs et de fonctions sur les points forts du territoire ( $c^{\prime}$ est bien la raison pour laquelle les pouvoirs publics français se refusent à bloquer le développement de la métropole parisienne, par crainte d'en affaiblir la compétitivité dans le cadre européen); d'autre part, la tendance croissante des pouvoirs politiques et surtout financiers à adopter les mêmes schémas de concentration géographique aux profits des principales métropoles que les états-majors des grandes entreprises (la réalité de la Déprise Régionale est en contradiction avec les discours sur l'aménagement du territoire).

Tout ceci conduit à une conclusion évidente: par le niveau de leurs fonctions, la variété de leurs activités, la diversification des qualifications et des savoir-faires, la richesse des équipements de tous ordres, leurs capacité à concentrer les informations les plus diverses... etc.., les villes et surtout les grandes villes constituent les milieux les plus aptes à répondre aux exigences actuelles de l'innovation et des activités technologiquement avancées. C'est vers les villes et surtout vers les grandes villes, celles qui offrent un maximum d'externalités et de possibilités de création de relations locales et régionales, que se réalisent ces concentrations sélectives qui marquent aujourd'hui l'espace géographique. De ce point de vue, le cas des métropoles est tout à fait exemplaire; nous l'aborderons plus loin.

Auparavant, une remarque s'impose: il existe une alternative à l'ajustement par les concentrations d'activités et par les migrations sélectives d'actifs; à savoir: l'ajustement par le transfert spatial des données réalisé grâce à l'outil informatique. Nous faisons ici, bien entendu, référence au formidable développement actuel du télétravail, qui permet aux entreprises et collectivités de réaliser de substancielles économies sur les coûts du travail (cf: la création et la dispersion régionale de centres de télésecrétariat au service de la métropole parisienne l'importance des traitements de données réalisés pour les pays européens par l'Inde, les Philippines, Taïwan ... etc.).

Les concentrations spatiales de fonctions et d'activités de haut niveau, ainsi que les concentrations d'actifs très qualifiés se réalisent, en priorité au bénefice des métropoles. Le phénomène métropolitain ne correspond pas à une simple notion d'importance démographique. La "métropole" c'est la grande ville qui concentre les fonctions de haut niveau ainsi que les pouvoirs décisionnels de nature diverse, c'est l'organisme urbain capable de mener simultanément des stratégies de contrôle de ses contenus (infrastructures et équipements, ressources, informations, main-d'oeuvre qualifiée, externalités de service...), des stratégies de relations inter-personnelles et de contacts directs (en tirant parti de la proximité spatiale), des stratégies d'innovation en continu (grāce à la juxtaposition immédiate des activités sophistiquées, des actifs qualifiés, des informations scientifiques et techniques). La 
"métropole" est donc, par excellence, le milieu adapté aux exigences de la flexibilité. Cependant, cette flexibilité et cette capacité d'innovation permanente ont une inter-face, qui "justifie" le caractère sélectif de ce type de milieu: la métropole est un milieu où les coùts d'implantation ainsi que les coûts de reproduction de la force de travail sont particulièrement élevés; et le poids des coûts pèse aussi bien sur les entreprises, qui doivent pouvoir les supporter mais aussi payer des salaires élevés à la main-d'oeuvre, que sur les habitants, qui doivent ètre en mesure, par leur niveau de revenus, de payer des loyers et des coûts immobiliers élevés. En tendances dominantes, la métropole est un milieu concentrant les activités générant une forte valeur ajoutée et employant une force de travail hautement qualifiée; pour cette dernière, la plupart des métropoles se distinguent par la mise en place de "viviers permanents" dans lesquels les entreprises puisent leur force de travail au fur et à mesure des besoins (ce qui permet de réguler le niveau des salaires et de maintenir la précarisation de l'emploi).

La combinaison des diverses caractéristiques qui précèdent permet de bien comprendre une autre particularité des métropoles, directement liée au phénomène de migrations sélectives: le milieu métropolitain est, par ses exigences, celui qui génère le plus d'exclusion sociale, de marginalisation, de ségrégation à la fois sociale et géographique (cf: le rejet des exclus en périphérie le transfert des activités banales vers les périphéries nationales ou internationales). Tout se passe donc comme si le milieu métropolitain fonctionnait simultanément comme une "pompe aspirante" pour les activités sophistiquées et les forces de travail très qualifiées, et comme une "pompe refoulante" pour les activités et les main-d'oeuvre banales.

En matière d'ajustement aux effets de la crise, la problématique proposée par les vieux bassins industriels d'Europe occidentale est bien différente; pourtant, on y retrouve le poids et le rôle des migrations sélectives. Domaine par ex- cellence des activités traditionnelles (charbonminessidérurgie-textile), les vieux bassins industriels d'Europe occidentale ont, depuis les années soixante, cumulé toutes les difficultés de l'époque contemporaine: déclin rapide des activités industrielles nées au XIXème siècle, obsolescence des savoir-faire et déqualification des emplois d'une main-d'oeuvre particulièrement abondante et souvent peu qualifiée, concurrence des pays du tiers monde pour le travail peu ou non qualifié, accumulation de crises sociales et urbaines liées à la crise de l'économie régionale... etc. Partout en Europe, ces vieux bassins sont particulièrement sensibles aux effets de la crise du système de production fordiste et tous ont bien du mal à répondre aux exigences nouvelles du système de production flexible. Dans toutes ces régions, une mème question se pose: comment sortir de la spirale de crises cumulatives alors que tout est à reconstruire, que le milieu n'est guère attractif, que la capacité de développement endogène est des plus limitées, que les greffes industrielles prennent difficilement... ? La réponse a presque toujours été la même: il faut attirer des activités sohistiquées et des actifs hautement qualifiés. Il n'est donc pas étonnant de constater que les vieilles régions industrielles en reconversion (Nord, Lorraine, Saint-Etienne, Ruhr, Borinage, Midlands, Tyneside ... etc.) constituent, hors des espaces métropolitains, le second espace d'accueil des technopoles. Mais alors, les migrations sélectives ne sont plus seulement sociales ou économiques, elles sont aussi géographiques parce que ces technopoles se localisent étroitement en lisière d'espaces métropolitains (cf: Villeneuve d'Asq et la métropole lilloise Dorstfeld et l'agglomération de Dortmund... etc.) alors que les activités régionales dominantes se localisaient auparavant sur les bassins houillers-miniers puis dans les zones industrialo-portuaires.

La ségrégation qualitative des emplois, et l'ajustement géographique par les migrations sélectives, ne procèdent pas uniquement de la métropolisation et de l'innovation technologi- 
que; les deux sont considérablement renforcées par les effets spatiaux de la mise en place des systèmes de transport à grande vitesse (de type: réseau ferroviaire TGV). De même que les échangeurs d'autoroutes, les gares TGV, les gares d'interconnection ainsi que les plateformes multimodales offrent aux entreprises une formidable rente de localisation, en permettant l'accés direct aux transports rapides. Ces lieux, particulièrement attractifs, voient surtout s'implanter des activités modernes aptes à payer des coûts de localisation élevés; ils attirent également une main-d'oeuvre qualifiée du fait de leur appartenance au monde des activités technologiquement avancées. Ces points forts du territoire sont donc, indirectement, générateurs de migrations sélectives liées aux facilités d'accès aux transports rapides. Finalement, la métropolisation, l'innovation technologique et les transports à grande vitesse, qui expriment les réactions "spontanées" à la crise, concourent à accentuer les ségrégations qualitatives dans l'espace. En est-il de même pour ce qui concerne les actions de l'Etat et des collectivités?

\section{Les strategies \\ territoriales publiques}

Le passage du système de production fordiste au système de production flexible a imposé l'obligation de diffuser l'information et de transférer les technologies dans l'espace géographique. Tous les acteurs du territoire s'y emploient, à des degrés divers. Le rôle des différents acteurs n'est guère compréhensible si on ne tient pas compte de cette double obligation.

Les différences étant nettement marquées entre les pays européens, nous nous réfèrerons au seul exemple français pour l'étude des actions territoriales de l'Etat et des diverses collectivités publiques.

L'Etat est, bien entendu, garant des équilibres territoriaux et des égalités sociales; mais il est aussi garant de la compétitivité de l'économie au plan international. Il est vrai que, au moins au niveau du discours, la lutte contre les déséquilibres inter-régionaux est un principe fondamental des politiques d'aménagement du territoire; il est encore plus vrai que l'Etat n'a pu éviter, sans doute du fait de la globalisation, que la rentabilité des activités et des localisations devienne le paradigme majeur des politiques d'aménagement. Il est donc toujours nécessaire de bien distinguer le contenu du discours politique et la réalité des politiques mises en place: en pratique les pouvoirs publics, de mème que les entreprises privées, cherchent à renforcer les points forts du territoire, ils participent donc directement aux concentrations géographiques qui s'opèrent aux profits des métropoles-technopoles-axes de transports rapides. L'ambivalence caractérise de façon permanente l'action de l'Etat à l'égard des territoires. Les politiques de décentralisation industrielle, puis tertiaire, puis administrative, qui se sont succédées au fil des années entre la décennie cinquante et le début des années quatrevingt, ont incontestablement concouru à une meilleure répartition territoriale des activités et des hommes. Si les résultats acquis alors ne sont guère discutables au plan quantitatif, des emplois ont été créés et des compétences publiques ont été transférées en province, le bilan en termes qualitatifs prête le flanc à la critique car les mouvements de concentration géographique des fonctions et des activités sophistiquées, de mème que la déprise régionale des pouvoirs de commandement, n'ont pas cessé au bénéfice de la métropole parisienne et des métropoles de province (d'où le sentiment, fréquent en France, que l'aménagement du territoire a surtout joué Paris contre la province, pour des raisons évidentes de compétitivité dans le cadre européen !). Cependant, à la suite des crises de la décennie soixantedix, l'Etat développe deux politiques spécifiques en faveur du territoire, politiques susceptibles de conduire à de meilleurs équilibres inter-régionaux.

C'est, en premier lieu, la politique très volontariste de diffusion spatiale de l'information dont l'objectif est de donner à tous, quel que soit le lieu d'implantation, des possibilités d'accès aux 
informations économiques-scientifiques-techniques. Cette action s'appuie tout à la fois sur des organismes spécifiques, comme les "Agences Régionales à l'Information Scientifique et Technique" (ARIST. 1978) ou encore les antennes régionales de "l'Agence Nationale pour la Valorisation de la Recherche" (ANVAR. 1980), et sur la création de réseaux territoriaux de télécommunications pour la diffusion des données (TRANSPAC.1978) ou des images (TRANSFAX. 1983). C'est, en second lieu, un effort considérable réalisé par les pouvoirs publics pour assurer le transfert technologique, à la fois dans l'ensemble de la structure économique et sur l'ensemble du territoire. L'objectif visé étant ici de permettre à toutes les entreprises, quelle que soient leur taille et leur localisation, d'accéder à l'innovation technologique (cf: les "Délégués Régionaux à la Technologie" (1980) "Les Centres Régionaux d'Innovation et de Transfert Technologique" (1982) le "Fonds de la Recherche et de la Technologie" (1988)J. Comment expliquer alors la permanence des concentrations sélectives au profit des points forts du territoire? Il ne peut y avoir de réponse sûre à cette question, nous nous limiterons donc à des hypothèses. Le passage du fordisme à la flexibilité s'est accompagné, d'une part, de la fin de l'Etat-providence, d'autre part, de l'émergence du modèle de développement par le bas, enfin, d'un réel retrait territorial de l'Etat en faveur des collectivités régionales et locales. Si on ajoute à ce nouveau contexte les effets de la mondialisation, on comprend que les collectivités de tous niveaux sont désormais en concurrence directe pour ce qui concerne les facteurs de développement localisé; et comme toujours en matière de concurrence, les forces nouvelles s'accumulent sur les points forts du territoire, des points qui tendent à devenir de plus en plus forts. Cela signifie que le système de production et le modè-le de développement aujourd'hui dominants sont en contradiction avec les objectifs affichés de l'aménagement du territoire.

Dans le cadre de cette concurrence rendue plus acharnée par la crise, le problème posé aux collectivités régionales et locales est, apparemment, simple: il faut attirer les activités modernes et technologiques car elles représentent l'assurance d'avoir des ressources financières, de voir se créer des emplois nouveaux, de provoquer l'essor urbain. Et on peut dire que partout, la stratégie d'attraction repose sur la même "politique de la carotte" qui consiste à mettre à la disposition des entreprises les externalités, les équipements, les facilités financières et fiscales, les aides diverses dont elles ont besoin pour fonctionner dans de bonnes conditions de compétitivité. Mais la "carotte" n'est pas de même taille dans une agglomération millionnaire, dans une petite ville régionale ou dans l'une de ces milliers de communes françaises qui comptent encore moins de 1.000 habitants! Autrement dit: il y a tout lieu de penser que les ségrégations qualitatives et les migrations sélectives, modes de réponse à la crise, iront en s'accentuant.

Finalement: les territoires, au plan qualitatif, connaissent une évolution duale dans laquelle s'opposent des espaces très attractifs et des espaces marginalisés et plus ou moins "laissés pour compte" Faut-il voir là un résultat inévitable de la crise et du nouveau système de production?

\section{Bibliografia}

AYDALOT, P.. "La division spatiale du travail" In : PAELI NCK et SALLEZ(eds). Espace et localisation. Paris. Economica, 1983.

BERNARDY, M. \& BOISGONTIER, P.. La technopole.
Paris. L'Harmattan, 1996.

BOUREILle, B. \& GUESNIER, B. (sidd). Dynamique des activités et évolution des territoires. Poitiers. ADICUEER, 1994. 
BRUNET, R. \& SALLOIS, J. (sidd). France. Les dynamiques du territoire. Montpellier. DATAR et Gip RECLUS, 1986.

CASTELLS, M. (ed). High technology, space and society. Beverly Hills. Sage, 1985.

FEDERWISC, H. J. \& ZOLLER, H.G. (sidd). Technologies nouvelles et ruptures régionales. Paris. Economica, 1986.

FISCHER, A.. Industrie et espace géographique. Paris. L'Harmattan, 1994.

FISCHER, A. \& MALEZIEUX, J. (sidd). Industrie et aménagement. Paris. L'Harmattan, 1999.

MALECKI, E.. Technology and economic development. New York. Wiley, 1992.
MASSEY, D.. Spatial division of labour. Social structures and the geography of production. Londres. Macmillan, 1984.

PECQUEUR, B. (ed). Dynamiques terri-toriales et mutations économiques. Paris. L'Harmattan, 1996.

SAVY, M. \& VELTZ, P. (sidd). Les nouveaux espaces de l'entreprise. La Tour d'Aigues. Ed. de l'Aube/DATAR, 1993.

SCOTT, J. A. \& STORPER, M. (eds). Production. Work. Territory. Winchester. Unwin Hyman. THIREAU, V. 1993 Les nouvelles dynamiques spatiales. Paris. L'Harmattan, 1986.

VELTZ, P. Mondialisation, villes et territoires. L'économie d'archipel. Paris. PUF, 1996. 